\title{
The ALEXIS Point Source Detection Effort
}

\section{DIANE ROUSSEL-DUPRÉ, ${ }^{1}$ JEFF BLOCH, ${ }^{1}$ SEAN RYAN, ${ }^{1}$ BRADLEY EDWARDS, ${ }^{1}$ TIMOTHY PFAFMAN,${ }^{1}$ KERI RAMSEY, ${ }^{2}$ AND STEVE STEM ${ }^{1}$}

\author{
${ }^{1}$ Astrophysics and Radiation Measurements Group, Los Alamos National Laboratory, \\ P.O. Box 1663, MSD436, Los Alamos, New Mexico 87545 USA \\ ${ }^{2}$ Space Data Systems Group, Los Alamos National Laboratory, P.O. Box 1663, MSD440, \\ Los Alamos, New Mexico 87545 USA
}

Los Alamos National Laboratory's $A L E X I S$ satellite (a wide area EUV monitoring instrument) was launched April 25, 1993. Due to the damage sustained at launch by the satellite, the $A L E X I S$ project team has had to spend over a year devising new methods to determine spacecraft attitude knowledge, essential for putting photons back on the sky correctly. These efforts have been successful and currently the $A L E X I S$ attitude solutions are precise to better than 0.5 degree close to the original 0.25 degree pre-flight specification. This paper will discuss the number and types of point sources that have been revealed in the $A L E X I S$ data to date. We will also discuss $A L E X I S$ observations of the June, 1994 super outburst of the Cataclysmic Variable VW Hyi, a program to look for simultaneous EUV emission from Gamma Ray Bursts, as well as an effort to detect EUV transients with a $12-24$ hour response time.

\section{Introduction}

Los Alamos National Laboratory's $A L E X I S$ satellite, containing six EUV telescopes was launched April 25, 1993. ALEXIS is a sky monitor/survey experiment, and with each 50 second rotation, the satellite's six wide field of view telescopes scan nearly half of the sky. The telescopes have narrow energy responses centered at 66,73 and $93 \mathrm{eV}$. They are arranged in binocular pairs which are arranged to look (1) along, (2) $45^{\circ}$ to and (3) $90^{\circ}$ to what was to suppose to be the spin axis. The six telescopes are tuned to 3 different wavelengths.

\section{Attitude Solutions and Point Source Calibration}

Due to the damage sustained to the magnetometer at launch which invalidated most of the preflight analysis software and on-board attitude control algorithms (Bloch 1995), the $A L E X I S$ project team has had to (1) learn to steer the satellite from the ground and (2) spend over a year devising new methods to determine spacecraft attitude knowledge, essential for putting photons back on the sky correctly. Part of this effort was to use the data from bright sources (HZ43 primarily) to determine the individual bore sight corrections required for each new version of the attitude solutions. These efforts have been successful and currently the $A L E X I S$ attitude solutions are precise to better than 0.5 degrees, which is the minimum requirement to be able to detect weak sources in the sky maps. The first $A L E X I S$ sky maps that contained substantial amounts of coadded data were produced on November 4,1994 , which revealed several point sources in addition to the bright sources HZ43 and the Moon which had been detected in earlier, less sophisticated processing.

The first effort in identifying point sources entailed using our first generation processed sky maps to validate the attitude solutions. To do this, we made 10 day composite sky maps, looked for point sources within these maps and then plotted as a function of time 
TABLE 1. Previously identified sources found in the ALEXIS data

$\begin{array}{lrrl}\text { Name } & \text { RA }\left({ }^{\circ}\right) & \text { Dec }\left({ }^{\circ}\right) & \text { Spec. Type } \\ \text { WD0050-332 } & 12.74 & -33.29 & \text { DA1 } \\ \text { RE2214-491 } & 33.64 & -49.19 & \text { DA } \\ \text { Feige 24 } & 38.44 & 3.58 & \text { DA0 } \\ \text { V471 Tau } & 57.54 & 17.18 & \text { DA2 } \\ \text { VW Hyi } & 61.60 & -71.05 & \text { CV } \\ \text { MCT0455-2812 } & 73.97 & -28.05 & \text { DA } \\ \text { G191-B2B } & 76.41 & 52.94 & \text { DA0 } \\ \text { WD0549+158 } & 88.21 & 15.67 & \text { DA1 } \\ \text { WD0642-166 } & 101.12 & -16.86 & \text { DA2 } \\ \text { U Gem } & 118.05 & 22.00 & \text { CV } \\ \text { RE1032+532 } & 157.80 & 53.41 & \text { DA } \\ \text { WD1254+223 } & 194.27 & 22.01 & \text { DA1 } \\ \text { HZ43 } & 199.23 & 29.19 & \text { DA1 } \\ \text { WD1501+55 } & 222.54 & 66.39 & \text { DZ0 } \\ \text { WD2111+498 } & 318.04 & 49.94 & \text { DA2 } \\ \text { RE2156-543 } & 329.62 & -54.35 & \text { DA } \\ \text { WD2309+10 } & 348.04 & 10.98 & \text { DA0 } \\ \text { WD2309+105 } & 348.07 & 11.0 & \text { DA0 }\end{array}$

the positional offsets looking for systematics. We have done this for HZ 43, G191-B2B, RE2214-49, and MCT0455-2812. Only HZ43 and G191-B2B were visible in all three telescope pairs. For the initial data processing, the histogram distribution of offsets was double peaked with a peak at 0.25 and 0.45 degrees. The peak at 0.45 was due to under determined bore sight correction terms for telescope pair 2 (TP2) and TP3 which have recently been corrected with the addition of several months more data. Currently, point sources observed in TP2 and TP3 have offsets of order 0.25 or less.

To determine point source locations, we first create standard deviation maps and Lampton fluctuation maps (Lampton 1994), which improves detection at low count rate levels, and search for point sources using the IDL "FIND" routine, and/or more recently use a parameter estimation procedure developed by Cash (1978). The on-line data bases for EUVE, ROSAT, Yale Bright Star, Gliese, and White Dwarf catalogs are then searched for likely candidates. Two of the detector filters have small pinhole leaks that allows $\mathrm{O}$ and B star UV radiation through, therefore, the Yale Bright Star catalog must be searched to assure that the source is a real EUV source. Recent changes to the data processing pipeline eliminate events associated with known pinholes. If these on line catalogs do not yield a likely candidate, we then use the WEB SKYVIEW page to search other catalogs.

We compiled a list of all sources found in the 10 day maps. We then added all files for 1 year into composite maps for each telescope and then all maps for each energy band and searched these for point sources. Lists of the point sources found are located in the following tables. The quick first pass through the data has yielded eighteen bright previously identified sources (Table 1) and five transients, three of which are unique to the ALEXIS data set (Table 2). It is also possible that the detection of V471 Tau should be included in the transient list, but more work will be required to determine when it was observed. Several unknown sources were also identified as having a lampton fluctuation significance of greater than $10^{-5}$ probability of random fluctuation which are potentially other transients. They will need further study before they are formally included in the final $A L E X I S$ source catalog. 
TABLE 2. Observed ALEXIS transients

\begin{tabular}{|c|c|c|c|c|}
\hline Name & $\mathrm{RA}\left({ }^{\circ}\right)$ & $\operatorname{Dec}\left({ }^{\circ}\right)$ & Spec. Type & Telescope \\
\hline VW Hyi & 61.60 & -71.05 & $\mathrm{CV}$ & $(1 \mathrm{~B}-73 \mathrm{eV})$ \\
\hline U Gem & 118.05 & 22.00 & $\mathrm{CV}$ & $(2 \mathrm{~A}-93 \mathrm{eV})$ \\
\hline ALEXIS J1114+430 & 168.66 & 42.73 & CV & $\begin{array}{l}(1 \mathrm{~B}-73 \mathrm{eV}) \\
(1 \mathrm{~A} / 2 \mathrm{~A}-93 \mathrm{eV}) \\
(2 \mathrm{~B}-66 \mathrm{eV})\end{array}$ \\
\hline $\begin{array}{l}\text { ALEXIS J1139-685 } \\
\text { ALEXIS J1644-302 }\end{array}$ & $\begin{array}{l}174.51 \\
251.23\end{array}$ & $\begin{array}{l}-69.9 \\
-3.21\end{array}$ & $\begin{array}{l}\text { unknown } \\
\text { unknown }\end{array}$ & $\begin{array}{l}(1 \mathrm{~B}-73 \mathrm{eV}) \\
(2 \mathrm{~B}-66 \mathrm{eV})\end{array}$ \\
\hline
\end{tabular}

TABLE 3. ALEXIS pre-flight and observed count rate

$\begin{array}{ccll}\text { Telescope } & \begin{array}{c}\text { Pre-flight } \\ \text { Estimates (c/s) }\end{array} & \begin{array}{c}\text { Observed } \\ (\mathrm{c} / \mathrm{s}, \mathrm{Jan} 94)\end{array} & \begin{array}{c}\text { Observed } \\ (\mathrm{c} / \mathrm{s}, \text { Mar 95) }\end{array} \\ \text { HZ43 } & & & \\ \text { 1A } & 0.12 & 0.02 \pm 0.015^{\dagger} & 0.025 \pm 0.015 \\ \text { 1B } & 0.49 & 0.46 \pm 0.05 & 0.55 \pm 0.05 \\ \text { 3A } & 0.18 & & 0.26 \pm 0.03 \\ \text { 3B } & 0.13 & & 0.18 \pm 0.02 \\ \text { G191-B2B } & & & \\ \text { 1B } & 0.057 & & 0.05 \pm 0.015\end{array}$

$\dagger$ due to mirror response much narrower than initially predicted

Since HZ43 has been observed for more than a year, it can be use to determine the long term stability of the $A L E X I S$ telescope response. Table 3 contains the HZ43 predicted and observed count rates. As can be seen, the recent TP1 count rates are consistent to within the 1 sigma error bars of the observations made more than a year ago. In addition, the observed count rates for HZ43 and G191-B2B are consistent with preflight estimates. Thus, we conclude that the $A L E X I S$ telescopes have retained full preflight sensitivity two years after launch.

\section{Transient Search}

Three methods are currently being used to detect EUV transients in the ALEXIS data: (1) archival search, (2) daily automated sky map searches, and (3) manual inspection of daily sky map. The archival searches to date have primarily used the 10 day sky maps. This study has produced the list of sources found in Table 3 as well as the super outburst of Cataclysmic Variable (CV) VW Hyi observed May/June 1994. Searches for transients on shorter time scales will be done as soon as the detector backgrounds are better understood. Real time transient searches were initially done daily by hand. ALEXIS J1114-430 was discovered in this manner in November, 1994. Starting 1 January, 1995 , these searches were automated, so now the 12,24 , and 48 hour sky maps are searched for point sources. Point source locations found in the data by search algorithms are sent via e-mail to the science team for evaluation. U Gem and ALEXIS 1139-685 (Roussel-Dupré et al. 1995a) were discovered in this mode. In addition, manual searches of the data are done to spot check the e-mail point source lists which recently produced ALEXIS J1644-032 (Roussel-Dupré et al. 1995b) a system that was just below threshold on 24 and 48 hour maps, but exceeded threshold in the 36 hour map. 
Of the transients observed by $A L E X I S$, three have been CV's and two are of unknown origin. Two of the three CV's, VW Hyi and U Gem, have been previously studied, but $A L E X I S$ affords a detailed complete light curve unlike some previous observations.

The complete super outburst light curve of VW Hyi was observed by $A L E X I S$ May 30-June 6, 1994. The optical light curve can be classified as a Bateson type S6 due to the decrease of $0.5 \mathrm{mag}$ a 1.5 days after peak brightness was observed. The $A L E X I S$ observations show the EUV radiation lagging the optical enhancement by 0.75 days similar to previous super outbursts (Pringle et al. 1987). C. Mauche of LLNL obtained $E U V E$ target of opportunity observations during the outburst, however, $24 \mathrm{hrs}$ of $E U V E$ observations were not started until 2.5 days after initial optical enhancement. The EUV enhancement observed by $A L E X I S$ continued exhibiting large fluctuations until 6 June at which time it turned off rapidly. The $A L E X I S$ light curve will be combined with the $E U V E$ results to try to piece together as much information as possible about the super outburst.

ALEXIS J1114-430 was first observed in outburst on 28 November, 1994 as the satellite field of view (FOV) drifted over the location of the source. The outburst lasted until 13 December, 1994 at which time the source dropped below the detector threshold. The light curve was quite flat topped throughout most of the outburst until the final decay very reminiscent of outbursts from SS Cyg (Jones \& Watson 1992) and U Gem (Long et al. 1995). A literature search revealed a recently identified CV, AR $\mathrm{UMa}$, within the error box. AR UMa, previously identified as an oxygen-rich, semiirregular system with period of 69 days (Jura \& Kleinmann 1992), has recently been reclassified by (Remillard et al. 1994) as an AM Her type system. Fortuitously, Remillard (private communication) obtained optical spectra of this source on 13 December, 1994 which showed enhanced emission thus confirming our identification of this source in outburst. The optical light curve from the roboscope at Indiana University (Honeycutt, Turner \& Robertson, 1995) for this time showed AR UMA to be in a 130+ day enhancement; the EUV outburst occurred in the middle of this enhancement. The first EUV observations occur eight days after a short duration 0.5 magnitude optical enhancement. Because the telescope drifted onto the AR UMa location after the optical enhancement there is no way of knowing what the maximum EUV brightness was at the time of optical peak. The optical emission from AR UMa was essentially constant during the $A L E X I S$ observed outburst.

ALEXIS J1139-685 and ALEXIS J1644-032 both have similar time signatures and look to be potentially a new type of transient. Both systems were observed to be in outburst for of order 24-36 hours. Target of opportunity observations were requested of $E U V E$ for these systems and in both cases, $E U V E$ was able to slew to target and observe these systems starting less than 24-36 hrs after maximum light. But in both cases, $24 \mathrm{hrs}$ of observations failed to detect the transients. Other potential transients have also been observed in the data with time scales of 12-36 hours. These systems are different than the ROSAT Wide Field Camera transient, 2RE J1255+266 Dahlem et al. (1995); if ALEXIS J1139-685 and ALEXIS J1644-032 had similar decay rates as 2RE J1255+266, EUVE should have been able to easily detect the transients in $24 \mathrm{hrs}$ of observation. Searches of the error boxes for counterparts have failed to produce good candidates for these systems. ARIEL $V$ reported the identification of fast-transient Xray sources in their archive data (Pye \& McHardy 1983). They observed the transients to be essentially isotropic in distribution and predict that the rate should be about one per 3 days. The average duration of the X-ray fast transients was typically a few hours. Although preliminary estimates for the number of fast transients observed by $A L E X I S$ appear to be similar to those observed by $A R I E L V$, the $A L E X I S$ transients are of longer 
duration than those observed by $A R I E L V$ and thus might constitute a different class of objects.

An effort is also underway to search the $A L E X I S$ archive for EUV detections of gamma ray bursts (GRB). An automated program takes the $A L E X I S$ photon list and checks to see if the detectors were on, at the correct high voltage setting, not looking at the earth, etc. during times of the BACODINE GRB triggers. The majority of the time the detectors have been off, but for a hand full of events for which the telescopes were in proper configuration, no clear signature of GRB events were present in the data. This effort continues with searches pre and post burst for weak emission within the error boxes.

\section{Conclusion}

As $A L E X I S$ begins its third year on orbit with all of the problems of the launch failure now resolved, the full potential of the monitoring capability of $A L E X I S$ is starting to be realized. Especially exciting is the complete coverage of $\mathrm{CV}$ outburst light curves and the detection of fast transients.

This effort is supported by the US Department of Energy, but would not be possible without the dedicated efforts of the entire $A L E X I S$ team.

\section{REFERENCES}

BLOCH, J. J. 1995, EUV Astrophysics with ALEXIS: The Wide View, these proceedings

CASH, W. 1978, Parameter Estimation in Astronomy through Application of the Likelihood Ratio, ApJ, 228, 939

Dahlem, M., Krysing, H. -C., White, S., Engels, D., Condon, J. J., \& Voges, W. 1995, RE J1255+266: Detection of an extremely bright EUV transient, A\&A, 295L, 13

Honeycutt, K., Turner, G. W., \& Robertson, J. 1995, RoboScope's Data Archive, World Wide Web http://www.astro.indiana.edu/apt/

HwANG, L. -S. \& TUCK, E. O. 1992, The EXOSAT Observations of SS Cygni, MNRAS, 257, 633

JuRA, M. \& KLEInMANN, S. G. 1992 1992, Oxygen-Rich Semiregular and Irregular Variables, ApJS, 83, 329

Lampton, M. 1994, Two-sample Discrimination of Poisson Means, ApJ, 436, 784

Long, K. S., MaUche, C. W. Szkody, P., \& MatTeI, J. A. 1995, EUVE Observations of U Gem, in Proc. of the Padova-Abano Conference on Cataclysmic Variables, in press

Pringle, J. E., Bateson, F. M., Hassall, B. J. M., Heise, J., van der Woerd, H., Holberg, J. B., Polidan, R. S., van Amerongern, S., van Paradijs, J., \& Verbunt, F. 1987, The Ariel V sky survey of fast-transient X-ray sources, MNRAS, 225, 73

Pye. J. P., \& McHARDY, I. M. 1983, The Ariel V sky survey of fast-transient X-ray sources, MNRAS, 205, 875

Remillard, R. A., Schachter, J. F., Silber, A. D., \& Slane, P. 1994, 1ES 1113+432: Luminous, Soft X-ray Outburst from a Nearby Cataclysmic Variable AR Ursae Majoris, ApJ, 426, 288

Roussel-Dupré, D., Bloch, J. J., Edwards, B. C., Pfafman, T. E., Priedhorsky, W. C., Ryan, S., Smith, B. W., Siegmund, O. H. W., Cully, S., Rodriguez-Bell, T., Vallerga, J., \& Warren, J. 1995a, ALEXIS J1139, IAU Circ. No. 6152

Roussel-Dupré, D. \& The ALEXIS Team 1995b, ALEXIS J1644, IAU Circ. \#6170 